\title{
Multidisciplinary Approach to Management of Hypofibrinogenaemia in Pregnancy, a Case Report
}

\author{
Slagjana Simeonova Krstevska, ${ }^{1}$ Elizabeta Todorovska, ${ }^{2}$ Tatjana Makarovska Bo- \\ jadjieva, ${ }^{2}$ Elena Petkovic, ${ }^{2}$ Saso Stojcevski, ${ }^{1}$ Igor Samardziski, ${ }^{1}$ Saso Spasovski, ${ }^{1}$ \\ Violeta Dejanova, ${ }^{2}$ Radica Grubovic, ${ }^{2}$ Florije Raka, ${ }^{2}$ Viktorija Jovanovska, ${ }^{1}$ Irena \\ Todorovska, ${ }^{1}$ Vesna Livrinova, ${ }^{1}$ Aneta Sima, ${ }^{1}$ Sasa Jovcevski, ${ }^{1}$ Daniel Milkovski ${ }^{1}$
}

\begin{abstract}
Inherited fibrinogen disorders introduce risk for recurrent abortions, sub-chorionic haematoma, placental abruption and postpartum haemorrhage. This is a case report of a successful pregnancy outcome in a 37-year old woman with hypofibrinogenaemia. She was referred to a coagulation test in the first trimester because of history of preeclampsia and HELLP syndrome in previous pregnancy. Hypofibrinogenaemia was diagnosed with fibrinogen level of $0.7 \mathrm{~g} / \mathrm{L}$. During the pregnancy she was regularly monitored for fibrinogen levels and multiple cryoprecipitate concentrates were given. She delivered at 39th gestation week, with elective caesarean section under general anaesthesia. There was one episode of postpartum haemorrhage treated with 2 units of red blood cells, repeated infusions of cryoprecipitate to obtain the level of fibrinogen of $2 \mathrm{~g} / \mathrm{L}$. She was discharged on the 6th postpartum day in a good condition. In these disorders levels of fibrinogen should be higher than $1 \mathrm{~g} / \mathrm{L}$ during pregnancy or $2 \mathrm{~g} / \mathrm{L}$ in case of caesarean section for successful prenatal and peripartal management.
\end{abstract}

Key words: Hypofibrinogenaemia, pregnancy, peripartal management.
(1) University Clinic for Obstetrics and Gynaecology, Skopje, North Macedonia. 2) Institute for Transfusion Medicine, Skopje, North Macedonia.

Correspondence:

SLAGJANA SIMEONOVA KRSTEVSKA

E: simeonovas@yahoo.com

\section{ARTICLE INFO}

Received: 9 May 2020

Revision received: 7 June 2020 Accepted: 9 June 2020

\section{Introduction}

The coagulation system has one final goal: convert fibrinogen to fibrin and form a clot. During pregnancy changes in the system of coagulation and fibrinolysis occur. Thrombin and fibrin generation increase to facilitate placental implantation and prevent excessive haemorrhage during delivery. Normal pregnancy causes progressive increase in maternal plasma D-dimer concentration from conception until delivery. ${ }^{1}$

Fibrinogen (normal concentration in blood between 2.0 and $4.5 \mathrm{~g} / \mathrm{L}$ ) supports pregnancies by maintaining haemostatic balance and stabilising the foetal-maternal junction and embryo implantation. ${ }^{2}$ Maternal fibrinogen and factor XIII are essential after 4-5 gestational week in decidual stroma. ${ }^{3}$ Fibrinogen plays a positive role in the process of implantation. Coagulation changes of pregnancy help in regulating attachment of placenta and stabilising some placental detachments that may happen. Low fibrinogen may cause rupture affecting embryonic trophoblast infiltration and leading to haemorrhage. ${ }^{4}$

Inherited fibrinogen disorders introduce risk for: menorrhagia, menometrorrhagia; recurrent foe- 
tal loss, spontaneous abortions, bleeding in early gestations, sub-chorionic hematoma, placental abruption; increased incidence and severity of postpartum hemorrhage. ${ }^{5}$ There are two types of hereditary fibrinogen abnormalities: quantitative defects like afibrinogenaemia or hypofibrinogenaemia and qualitative defects like dysfibrinogeneamia or hypodisfibrinogenaemia (HD). First reported in 1935, prevalence of afibrinogenemia is extremely rare, around $1 / 1,000,000$. Prevalence of hypofibrinogenaemia is hard to say as there are many asymptomatic patients. ${ }^{6}$

Congenital defects of fibrinogen are caused by mutations in the FGA, FGB, or FGG genes, located on the $\mathrm{q}$ arm of 4 th chromosome at position 31.3. Hypofibrinogenaemia and dysfibrinogenaemia are autosomal dominant or recessive. There are more than 100 fibrinogen mutations that have been associated with hypofibrinogenaemia. ${ }^{7} \mathrm{Hy}-$ pofibrinogenaemia could be: a) mild (fibrinogen levels $>1 \mathrm{~g} / \mathrm{L}$ ): asymptomatic, excessive bleeding can occur after injuries, surgery or delivery; b) moderate (fibrinogen levels between 0.1-1 g/L): bleedings can be spontaneous or caused by injuries, surgery, or delivery; c) severe (undetectable clot): spontaneous, severe, and even life-threatening bleeding may occur. ${ }^{8}$

Dysfibrinogenaemia results in abnormal fibrinogen function. It can be asymptomatic in $55 \%$ of the cases, can cause haemorrhage in $25 \%$ and thrombosis in $20 \% .^{9}$ Dysfibrinogenaemias are result of defect of fibrinogen molecule which impairs polymerisation of fibrin associated with irregular cross linking with factor XIIIa. Dysfibrinogenaemias can be associated with hypofibrinogenaemia and congenital trombophylia. ${ }^{10,11}$ As first such case was diagnosed in 1958 until today over 100 mutations have been reported. Dysfibrinogenemia increase the risk of spontaneous abortions, placental abruption, thrombosis and haemorrhage. ${ }^{12}$

If possible, during pregnancy fibrinogen levels should be more than $1 \mathrm{~g} / \mathrm{L}$ and in case of caesarean section $2 \mathrm{~g} / \mathrm{L}$. Best choice for treatment of acute bleeding episodes are fibrinogen concentrates. ${ }^{13}$ If unavailable, cryoprecipitate (a fibrinogen-rich plasma fraction) should be used. ${ }^{14}$

\section{Case history}

A thirty-seven-year old woman went to a gynaecologist to perform a routine exam due to the new pregnancy (gravida 2, para 1). There were no haemorrhagic and thrombotic events in the personal or family medical history.

Her previous pregnancy resulted in premature labour in 30th gestation week by caesarean section due to HELLP syndrome under general anaesthesia. The newborn had a body weight of $1,300 \mathrm{~g}$ and length of $42 \mathrm{~cm}$. It was admitted and treated in ICU and had a good outcome.

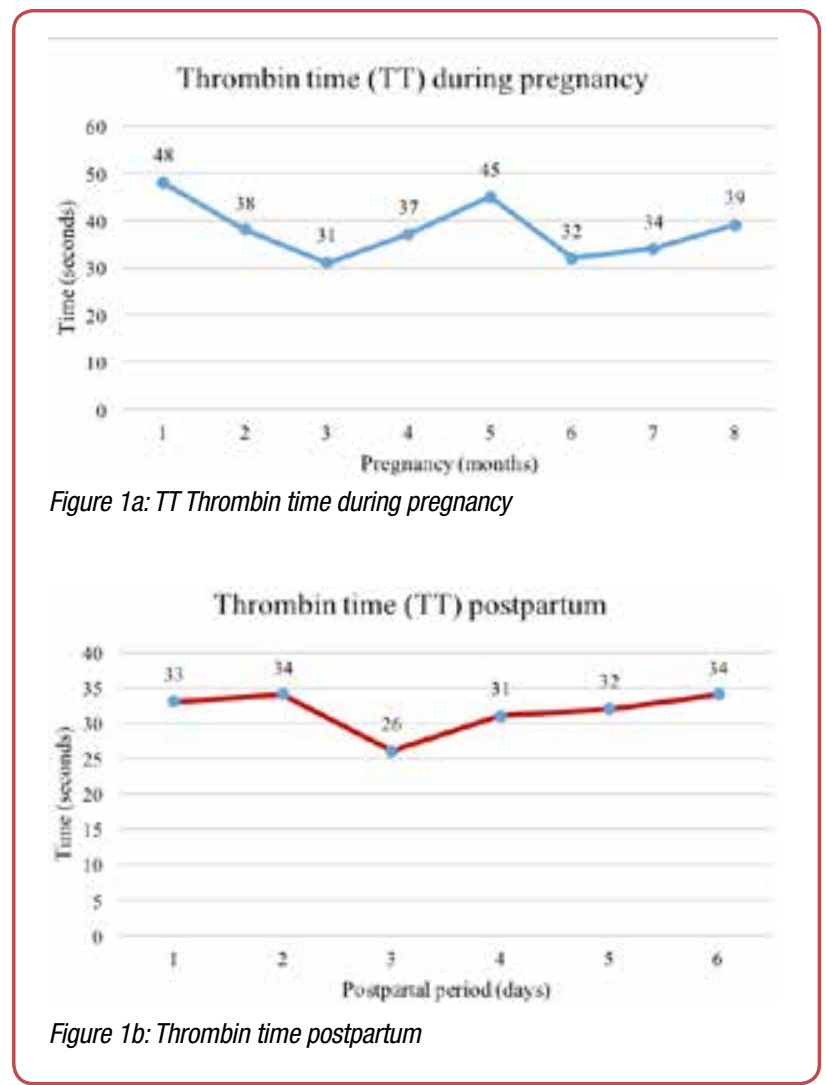

Figure 1: Thrombine time values during pregnancy and postpartum

During this pregnancy she was referred for a coagulation test in the 1st trimester (7th gestational week) when hypofibrinogenaemia was diagnosed with fibrinogen level of $0.7 \mathrm{~g} / \mathrm{L}$. Analysis for determining mutations/polymorphisms of the thrombophylic genes revealed: heterozygotic mutation of factor V H1299R, factor XIII V34L, PAI-1 5G/4G, MTHFR C677T, MTRR A66G polymorphisms.

Due to previous history of severe preeclampsia and HELLP syndrome, serum placental angiogen 
levels were analysed and risk for preeclampsia was ruled out (PLGF 103, sFLT 4708, sFLT 1/PLGF 4.6).

Non-invasive prenatal screening test was done due to maternal age and it came out as low risk for aneuploidy (chromosome 21, 18, 13, sex chromosomes, microdeletions).

Monitoring of fibrinogen levels on a two week or monthly interval was made by a specialist in transfusion medicine. She received multiple transfusions of cryoprecipitate (20 units each) during the 1st and 2nd trimester, as well as multivitamin supplementation with $\mathrm{B}_{6}, \mathrm{~B}_{12}$, methylfolic acid.

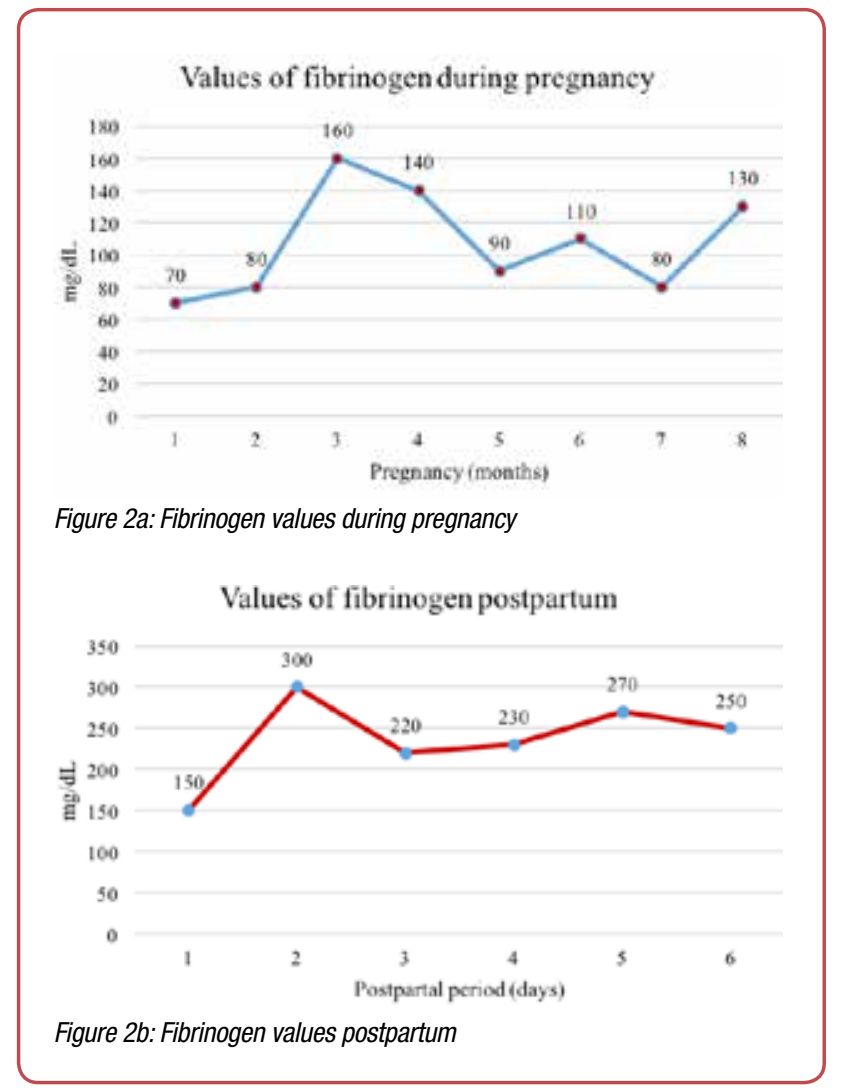

Figure 2: Fibrinogen level during pregnancy and postpartum

Platelet count ranged from $200 \times 10^{9} / \mathrm{L}$ at the beginning to $150 \times 10^{9} / \mathrm{L}$ at the end of pregnancy. Haematocrit was maintained above $30 \%$ during the pregnancy ( $42.7 \%-30.9 \%)$.

Screening haemostasis parameters were as follows: prothrombin time (PT) was in the normal range (12-14 s) during whole pregnancy; activated partial thromboplastin time (aPTT) was shortened according to the gestational age (29-24 s) and thrombin time (TT) was significantly prolonged (48-31 s) according to the cryoprecipitate substitu- tion (Figure 1a). During the pregnancy the level of fibrinogen varied from 0.7 to $1.6 \mathrm{~g} / \mathrm{L}$ according to the cryoprecipitate substitution (Figure 2a). D-dimers were increased according to the gestational age (from 227 to $2,900 \mathrm{ng} / \mathrm{ml}$ ).

Foetal growth was adequate for gestational age, placental echogenicity and doppler of the fetoplacental unit was closely monitored through the pregnancy by ultrasound. Pregnancy was uneventful with no signs of bleeding. Patient received thromboprophylaxis with enoxaparin in the late second and third trimester.

She was admitted at the University Clinic for Obstetrics and Gynaecology at 38th gestation week for adequate preparation for delivery. Anaesthesiology consultation was made with physical exam, ECG and cholinesterase analysis (history of prolonged wakening after general anaesthesia in previous caesarean section, results in reference values $6,389 \mathrm{U} / \mathrm{L}$ ).

Blood products were supplied: erythrocyte concentrate, fresh frozen plasma, cryoprecipitate. Thromboprophylaxis with enoxaparin was discontinued 12 hours before delivery. Cryoprecipitate infusion was administered with 10 units $/ 8 \mathrm{~h}$ interval until concentration of fibrinogen reached $3 \mathrm{~g} / \mathrm{L}$ before scheduling the caesarean section.

The patient was delivered at 39th gestation week by elective caesarean section under general anaesthesia. The newborn had weight of 3,550 g, length of $50 \mathrm{~cm}$ and Apgar score 9/10. Operative period was uneventful.

Postpartum screening haemostasis parameters were analysed daily: PT and APTT were in the normal range (12.1-13.2 s) as well as aPTT (2430.7 s), while D-dimers were decreasing. Platelet count ranged from 122 up to $361 \times 10^{9} / \mathrm{L}$. Postpartum TT and fibrinogen level is shown on Figure $1 \mathrm{~b}$ and $2 \mathrm{~b}$.

There was one episode of postpartal haemorrhage with estimated blood loss of $700 \mathrm{~mL}$ treated with standard uterotonics (oxytocin, methylergometrine, carboprost tromethamine). The haemoglobin levels were reduced from $114 \mathrm{~g} / \mathrm{L}$ to 74 $\mathrm{g} / \mathrm{L}$, haematocrit from 0.34 to 0.21 . She received two units of red blood cells (RBC), $3 \times 20$ units of cryoprecipitate (the level of fibrinogen was main- 
tained above $2 \mathrm{~g} / \mathrm{L}$ ), which improve haemoglobin up to $96 \mathrm{~g} / \mathrm{L}$ and haematocrit to 0.33 . Thromboprophylaxis with enoxaparin $2 \times 40 \mathrm{mg}$ was administered according to the protocol.

The patient was discharged from the clinic on the 6 th postpartum day in good condition.

\section{Discussion}

According to the genetic testing, dysfibrinogenemia was not confirmed in combination with the hypofibrinogenaemia in this case report. However, genetic testing revealed heterozygous mutation for FXIII V34L which is important for the interplay of FXIII and fibrinogen..$^{15}$

In pregnancy with hypofibrinogenaemia levels of fibrinogen should be more than $0.6 \mathrm{~g} / \mathrm{L}$ and higher than $1.5 \mathrm{~g} / \mathrm{L}$ at delivery. ${ }^{11}$ Even though case reports on this topic are few, pregnancy risk is connected to HD. Management of pregnancy with HD is appropriate to be on an individual basis considering the analysis, individual and family history. There have been case reports of good pregnancy outcome achieved with fibrinogen therapy 2-3 times a week or without any therapy..$^{13}$ Low molecular weight heparin prophylaxis can be administered in pregnancy and postpartum if there is a case of thrombotic phenotype.

As quantitative and qualitative fibrinogen disorders are rare, publication of cases like this is necessary. This patient had no previous haemorrhagic or thrombotic episodes in her lifetime. The previous pregnancy resulted in severe preeclampsia, so the coagulopathy was assumed to be connected to the HELLP syndrome. Nevertheless, this was the reason why coagulation testing was ordered in the very beginning of the second pregnancy.

Multidisciplinary management including specialist in transfusion medicine, haematologist, obstetrician/gynaecologist specialised in maternal-foetal medicine, anaesthesiologist and blood bank cervices is essential especially when fibrinogen concentrate is not available and antenatal care and postpartum haemorrhage treatment included cryoprecipitate. Combination of cryoprecipitate and anticoagulation helped the stability between the risk of bleeding and clotting in pregnancy.

The level of functional activity of fibrinogen below $0.7 \mathrm{~g} / \mathrm{L}$ is suggestive of dysfibrinogenaemia, which was not the case in this report. Was there the impact of the FXIII V34L heterozygous mutation on the fibrinogen function in combination with decreased fibrinogen level (not less than 0.7 $\mathrm{g} / \mathrm{L}$ ) as it was in this case, is hard to say. However, the cryoprecipitate transfusion enabled successful haemostasis and together with the absence of bleeding history, suggested that the genetic defect of FXIII had no influence on the haemostatic balance in this particular case. Having in mind that inherited fibrinogen disorders are very rare, further studies and more precise laboratory investigation are necessary for diagnostics of these conditions and for the prediction of obstetrics complications. ${ }^{13}$

\section{Conclusion}

Congenital fibrinogen disorders carry pregnancy risk, but it can be successfully managed by engaging the multifunctional team of specialists. As there are no randomised controlled studies, management is made on expert consensus. Successful perinatal outcomes can be accomplished by analysis of the fibrinogen levels and supportive therapy. Management must be individualised considering the personal history and the specific clinical situation. Interdisciplinary approach and close collaboration between the institutions is necessary since it was crucial to the positive outcome in this case.

\section{Acknowledgements}

None.

\section{Conflict of interest}

None. 


\section{References}

1. Clark P. Changes of hemostasis variables during pregnancy. Semin Vasc Med 2003 Feb;3(1):13-24.

2. Jarmila A. Zdanowicz, Daniel Surbek. Patient blood management in obstetrics - Review. Transfusion and Apheresis Science 2019 Aug;58(4):412-5.

3. De Moerloose P, Casini A, Neerman-Arbez M. Congenital fibrinogen disorders: an update. Semin Thromb Hemost 2013 Sep;39(6):585-95.

4. Casini A, de Moerloose P. Management of congenital quantitative fibrinogen disorders: a Delphi consensus. Haemophilia 2016 Nov;22(6):898-905.

5. Pritchard JA. Chronic hypofibrinogenaemia and frequent placental abruption. Report of a case. Obstet Gynecol 1961 Aug;18:146-51.

6. Hahn L, Lundberg PA, Teger-Nilsson AC. Congenital hypofibrinogenaemia and recurrent abortion. Case Report. Br J Obstet Gynaecol 1978 Oct;85(10):790-3.

7. Teraoka Y, Miyoshi H, Oshima K, Urabe S, Tanaka N, Kudo Y. Prenatal and peripartum management of patients with hypofibrinogenaemia resulted in two successful deliveries. Case Rep Obstet Gynecol 2017; 2017:9427359. doi: 10.1155/2017/9427359. Epub 2017 Feb 14.

8. Mensah PK, Oppenheimer C, Watson C, Pavord S. Congenital afibrinogenaemia in pregnancy. Haemophilia 2011 Jan;17(1):167-8.
9. Lebreton A, Casini A, Alhayek R, Kouteich KL, Neerman-Arbez M, de Moerloose P. Successful pregnancy under fibrinogen substitution in a woman with congenital afibrinogenaemia complicated by a postpartum venous thrombosis. Haemophilia 2015 Jan;21(1):e108-10. doi: 10.1111/hae.12584. Epub 2014 Dec 4.

10. Michael W. Mosesson. Dysfibrinogenemia and Thrombosis. Semin Thromb Hemost 1999; 25(3):311-319.

11. Yan J, Deng D, Cheng P, Liao L, Luo M, Lin F. Management of dysfibrinogenemia in pregnancy: a case report. J Clin Lab Anal 2018 Mar;32(3):e22319. doi: 10.1002/ jcla.22319. Epub 2017 Sep 26.

12. Munoz J, Schering J, Lambing A, Neal S, Goyert G, Green $\mathrm{PM}$, et al. The dilemma of inherited dysfibrinogenemia during pregnancy. Blood Coagul Fibrinolysis 2012 Dec;23(8):775-7.

13. Kobayashi T, Kanayama N, Tokunaga N, Asahina T, Terao T. Prenatal and peripartum management of congenital afibrinogenaemia. Br J Haematol 2000 May;109(2):3646.

14. Shapiro SE. Diagnosis and management of dysfibrinogenemia. Clin Adv Hematol Oncol 2018 Sep;16(9):602-5.

15. Kohler HP. Interaction between FXIII and fibrinogen. Blood 2013;121(11):1931-2. 